\title{
Selective Interference Alignment for MIMO Cognitive Femtocell Networks
}

\author{
Basak Guler, Student Member, IEEE, and Aylin Yener, Member, IEEE
}

\begin{abstract}
This paper presents a novel cross-tier interference management solution for coexisting two-tier networks by exploiting cognition and coordination between tiers via the use of agile radios. The cognitive users sense their environment to determine the receivers they are interfering with, and adapt to it by designing their precoders using interference alignment (IA) in order to avoid causing performance degradation to nearby receivers. The proposed approach judiciously chooses the set of users to be aligned at each receiver as a subset of the crosstier interferers, hence is termed selective IA. The proposed solution includes identification of the subspace in which cross-tier interference signals would be aligned followed by a distributed algorithm to identify the precoders needed at the selected interferers. The intra-tier interference is then dealt with using minimum mean squared error (MMSE) interference suppression. Numerical results demonstrate the effectiveness of selective IA for both uplink and downlink interference management.
\end{abstract}

Index Terms-Cognitive femtocells, heterogeneous networks, interference management, interference alignment.

\section{INTRODUCTION}

$\mathbf{F}$ EMTOCELLS are small base stations designed mainly for indoor use, to provide high data rates for next generation wireless cellular networks [1]. They are low cost plug and play devices purchased by the subscribers, providing coverage to a small area where they are installed [2]. Femtocell users (FU) utilize the internet backhaul, which reduces the load on the macrocell network, enabling the resources to be allocated to the truly mobile users. It is preferred for the femtocells to share the frequency band with the existing macrocell network, as the licensed band is highly populated, and frequency is a scarce resource. This fact, combined with the ad hoc deployment of femtocells, make cross-tier interference management challenging, and render centralized solutions less than practical.

Cognitive radios are software defined radios which are aware of their environment, and have the ability to learn from and quickly adapt to the variations in their environment and the network requirements by changing their transmission parameters [3], [4]. The need for cognitive radio emerged from the fact that current frequency allocations with fixed spectrum assignment do not utilize the frequency resources effectively [5]: Cognitive radios can monitor wireless transmissions and find transmission opportunities. In some cognitive scenarios, cognitive secondary users transmit only when they detect a

Manuscript received November 18, 2012; revised April 4, 2013 and June 26, 2013. The material in this paper was presented in part at the 2013 IEEE International Conference on Communications.

The authors are with the Department of Electrical Engineering, The Pennsylvania State University, University Park, PA, 16802, USA (e-mail: bxg215@psu.edu; yener@ee.psu.edu).

Digital Object Identifier 10.1109/JSAC.2014.140306 spectrum hole unused by primary users which have the property rights to the spectrum. Alternatively, cognitive users can coexist with the primary users, by adjusting their transmission power pattern so that the interference received by the primary users is not harmful [6]. This method has the advantage of utilizing the frequency band more efficiently [7], [8], provides inspiration for innovative uses of cognitive radios in emerging communication systems.

Cognitive radio principles have been used in tiered networks to sense the spectrum to allocate the available channels to users from different access points, enabling them to operate in the same frequency band [9]. The notion of cognitive femtocells was proposed to expand the femtocell/macrocell networks with abilities such as sensing, measuring, learning and adapting to their environment [10], [11]. Femtocell users are subscribed customers who, in addition to the macrocell service, are willing to pay a surcharge to use their femtocell access points for better indoor performance. Therefore, in order to encourage the indoor customers to install femtocells instead of using the macrocell network, wireless operators can consider giving priority to the femtocell users in terms of their QoS requirements. Motivated by this insight, we present a twotier network view that considers the femtocell users as primary users in the network, and the macrocell users as secondary users. However, we also note that as macrocell users need to have their connection as well, a model that enables both tiers to coexist and communicate in the same frequency band is necessary.

In this paper, we present a network design that tackles the interference management problem in a two-tier cognitive femtocell/macrocell network, where all femto and macrocell users transmit in the same band. In such a network, the uplink interference caused by the secondary (macrocell) users at the primary receivers (femtocell base stations) can be severe when the macrocell user (MU) is far from the macrocell base station (MBS)/secondary receiver (SR) and close to the femtocell base station (FBS), thereby transmitting with high power. We can combat this interference utilizing the adaptability of the radios and cross-tier cooperation. Conversely, in the downlink FBSs that a MU is close to can be detrimental to it, and the principle of adapting the transmissions is helpful.

As a means of effective interference management in the uplink, we propose to utilize interference alignment [12] for aligning the signals from the MUs that are causing high interference at multiple FBSs simultaneously. Interference alignment (IA) is shown to achieve the maximum number of degrees of freedom in a $K$-user interference channel [12] by aligning the interfering signals in a lower dimensional subspace at multiple receivers simultaneously. A number of 


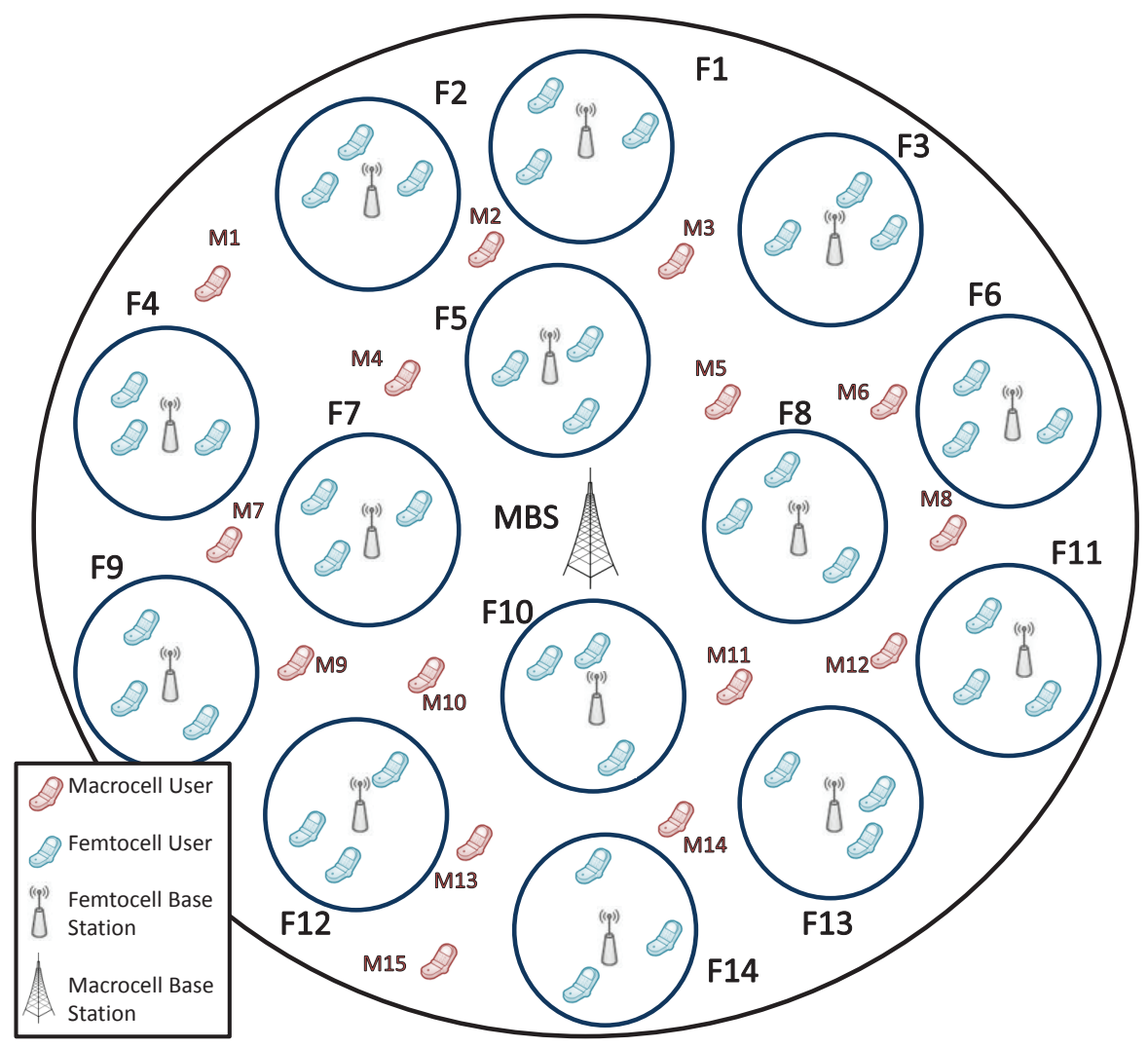

Fig. 1. System model for one MBS and multiple FBSs.

practical IA schemes have been developed to date, including minimizing the leakage interference [13], [15], maximizing the SINR [13], or minimizing MSE [14]. These algorithms are developed for single-tier $K$-user interference channels, in which each transmitter has an intended receiver, and the remaining transmitters are considered as interferers for that receiver. As an example, the minimum leakage interference/max SINR algorithms proposed in [13] use channel reciprocity and iterate between the receivers and transmitters [24] in order to minimize the leaked interference/maximize the SINR of the intended signal, respectively.

In the proposed two-tier system, just like in single-tier systems [12-16], the signals of the interferers (MUs) are restricted to a lower dimensional subspace received at each FBS. This in turn allows the FBS to use fewer receive antennas for canceling the macrocell interference, and to utilize the remaining degrees of freedom for improving the performance of the FUs. However, unlike the single-tier systems, spatial dimensions must be allocated in the best possible way to deal with macro and femtocell interference together. The proposed solution includes judicious selection of the macrocell interferers to align at multiple FBSs, and identification of the subspace in which cross-tier interference signals would be aligned followed by a distributed algorithm to identify the precoders needed at the selected interferers. The intra-tier interference, i.e., the interference from FUs is then dealt with MMSE interference suppression [23], [24].

We note that interference alignment for femtocell networks has recently been considered [17] with the objective of keeping the QoS (SIR) of macrocell users satisfactory, with no user selection. On the other hand, the approach proposed in this paper performs interference alignment with user selection, and is appropriately termed selective interference alignment. The IA methods proposed for $K$-user interference channels have been used in [18] and [19] for mitigating the intra-tier femtocell interference in the downlink of a split-frequency femtocellmacrocell network, in which macrocell and femtocells are assigned separate frequency bands. By contrast, our scheme considers the inter-tier interference management problem in a femtocell-macrocell network, aligning the interfering signals of one tier at the receivers of the other tiers.

Although the focus of this work is mainly on the uplink of this two-tier system, we also turn our attention to downlink, with the goal of identifying the advantages of cognition in managing the cross-tier interference in the downlink. We observe that by a mathematical approach similar to the uplink case, selective alignment can also prove useful for effectively managing interference from FBSs to MUs that are close to these FBSs.

The remainder of the paper is organized as follows: We introduce the system model in Section II. In Section III, we propose the MU selection for IA in the uplink. In Section IV, we present the distributed IA algorithm and its convergence. Precoder and decoder design for the femtocell users is described in Section V. Section VI provides the relevant discussion on our approach and is followed by the feasibility conditions in Section VII. Downlink scenario is analyzed in Section VIII. Numerical results are given in 
Section IX, demonstrating the advantage of selective IA. The paper is concluded in Section X. The following notation is used throughout the paper. Lower (upper) case bold letters represent vectors (matrices). $\mathbf{A}^{\dagger}$ refers to the pseudo-inverse of matrix A. $\mathbf{A}^{H}$ is used for the Hermitian transpose, and $\otimes$ for the Kronecker product. $\operatorname{tr}(\mathbf{A})$ is the trace of matrix $\mathbf{A}$. Finally, $|\mathcal{S}|$ denotes the cardinality of the set $\mathcal{S}$.

\section{SySTEM MOdEL}

The cellular network considered in Sections III-VII is the uplink of a cognitive co-existing macrocell-femtocell network with a single MBS/secondary receiver (SR) at the center and multiple FBSs/ primary receivers (PR) distributed over the macrocell coverage area, as shown in Fig. $1 .{ }^{1}$ We will consider the downlink scenario briefly in Section VIII. Each mobile user has $N_{t}$ transmit antennas. The number of MUs is denoted by $M$ and the number of FUs at the $f^{t h}$ FBS is denoted by $U_{f}$. The MBS and the $f^{t h}$ FBS have $N_{o}$ and $N_{f}$ receive antennas, respectively. The received signal at the $k^{t h}$ FBS is given as:

$$
\begin{aligned}
\mathbf{y}_{k}= & \underbrace{\sum_{i=1}^{U_{k}} \sqrt{p_{k i}} \mathbf{H}_{k k}^{i} \mathbf{W}_{k i} \mathbf{s}_{k i}}_{\begin{array}{c}
\text { signals from the users } \\
\text { of the } k^{\text {th }} \text { femtocell }
\end{array}}+\underbrace{\sum_{\substack{f=1 \\
f \neq k}}^{F} \sum_{u=1}^{U_{f}} \sqrt{p_{f u}} \mathbf{H}_{k f}^{u} \mathbf{W}_{f u} \mathbf{s}_{f u}}_{\begin{array}{c}
\text { interference received from } \\
\text { other femtocell users }
\end{array}} \\
& +\underbrace{\sum_{m=1}^{M} \sqrt{p_{\text {om }}} \mathbf{H}_{k o}^{m} \mathbf{W}_{\text {om }} \mathbf{s}_{\text {om }}}_{\begin{array}{c}
\text { interference received from } \\
\text { macrocell users }
\end{array}}+\mathbf{n}_{k}
\end{aligned}
$$

where $\mathbf{W}_{f u}$ denotes the $\left(N_{t} \times d\right)$ precoding matrix of the $u^{t h}$ user of the $f^{t h}$ femtocell, and $\mathbf{W}_{o m}$ represents the $\left(N_{t} \times d\right)$ precoding matrix of the $m^{\text {th }}$ MU.

The number of message bits transmitted from each mobile user is denoted by $d$. We assume the same number of bits are transmitted from each mobile user to simplify the analysis, noting that the results obtained in this paper can be extended to the case in which a different number of bits is transmitted from each user. $\mathbf{s}_{f u}$ is the $(d \times 1)$ message signal of the $u^{t h}$ user of the $f^{\text {th }}$ femtocell, and $\mathbf{s}_{\text {om }}$ represents the $(d \times 1)$ message signal of the $m^{\text {th }}$ MU. $\mathbf{H}_{k o}^{m}$ represents the channel from the $m^{t h}$ MU to the $k^{t h} \mathrm{FBS}$, and $\mathbf{H}_{k f}^{u}$ is the channel from the $u^{t h}$ user of the $f^{t h}$ femtocell to the $k^{\text {th }}$ FBS. The noise vector at the $k^{t h}$ FBS is denoted by $\mathbf{n}_{k}$, which consists of independent zero mean Gaussian random variables with $E\left\{\mathbf{n}_{k} \mathbf{n}_{k}{ }^{H}\right\}=\sigma^{2} \mathbf{I}$. Each element of the message signals $\mathbf{s}_{f u}$ and $\mathbf{s}_{o m}$ is chosen from $\{+1,-1\}$ randomly with equal probability for $u=1, \ldots, U_{f}, f=1, \ldots, F$, and $m=1, \ldots, M$. The power transmitted from the $f^{t h}$ user of the $u^{\text {th }}$ femtocell is $p_{f u}$, and the transmit power of the $j^{\text {th }} \mathrm{MU}$ is $p_{o j}$. We assume that the transmit powers of all users that belong to the same tier are fixed and equal. The precoding matrices of all mobile users satisfy $\operatorname{tr}\left(\mathbf{W}_{o j}^{H} \mathbf{W}_{o j}\right)=\operatorname{tr}\left(\mathbf{W}_{k u}^{H} \mathbf{W}_{k u}\right)=1$, $\forall k \in\{1, \ldots, F\}, u \in\left\{1, \ldots, U_{k}\right\}, j \in\{1, \ldots, M\}$. Although the focus of this work is cross-tier interference management, we provide a comprehensive solution in which

\footnotetext{
${ }^{1}$ We treat inter-macrocell interference as noise and concentrate on one macrocell.
}

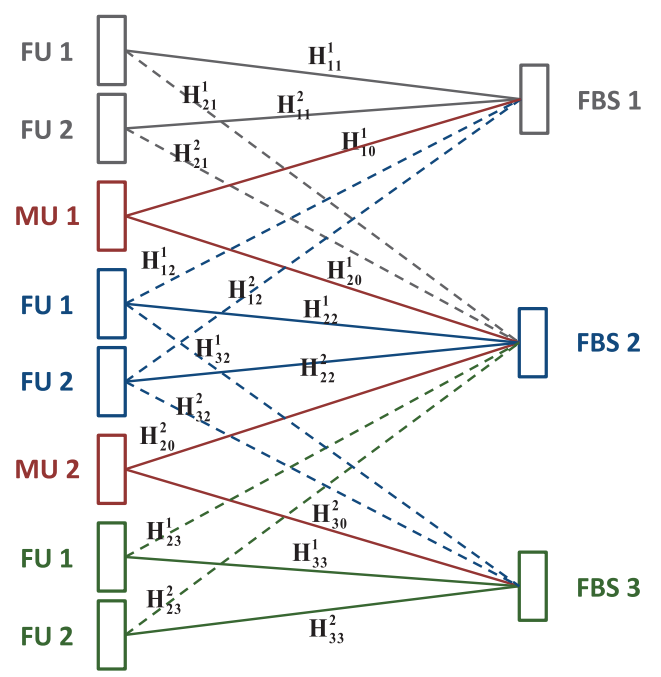

Fig. 2. Channel model for 3 FBSs, with 2 FUs in each femtocell, and 2 MUs.

femtocell-to-femtocell interference (interference from other femtocells) is dealt with MMSE interference suppression. An example system model with 3 FBSs and 2 MUs is depicted in Fig. 2, where the first MU is assumed to be causing high interference at FBSs 1 and 2, and the second MU is assumed to be causing high interference at FBSs 2 and 3 .

\section{Cognitive Macrocell User Selection}

In this section we describe how to determine the set of secondary/macrocell users to be aligned at each primary receiver/femtocell base station. Our system model considers a cognitive femtocell network where the femtocell base stations/primary receivers (PR) have the ability to listen and sense the secondary users (SU) transmitting nearby. We first note that the maximum number of users that can be aligned at each primary receiver is limited by the number of antennas, i.e., the spatial dimensions available for IA. Consequently, aligning all SUs at all PRs simultaneously is not feasible for typical user loads. Instead of attempting to align the entire set of SUs at every PR simultaneously, we propose a user selection algorithm in which only the dominant SUs, i.e., the SUs that cause the highest interference, are aligned at each PR. The user selection process starts with determining the $\mathrm{SU}$ that is causing the highest interference at each PR. Then, we define the set of dominant SUs at PR $k$ as $\mathcal{S}_{k}=\left\{\mathcal{S}_{k}^{1}, \mathcal{S}_{k}^{2}, \ldots, \mathcal{S}_{k}^{\left|S_{k}\right|}\right\}$, where:

$$
\begin{aligned}
\mathcal{S}_{k}^{1}= & \underset{j \in\{1, \ldots, M\}}{\arg \max } \operatorname{tr}\left(\left(\mathbf{H}_{k o}^{j}\right)^{H} \mathbf{H}_{k o}^{j}\right) \\
\mathcal{S}_{k}^{i}= & \left\{j \in\{1, \ldots, M\}: \operatorname{tr}\left(\left(\mathbf{H}_{k o}^{j}\right)^{H} \mathbf{H}_{k o}^{j}\right) \geq\right. \\
& \left.\tau \operatorname{tr}\left(\left(\mathbf{H}_{k o}^{S_{k}^{1}}\right)^{H} \mathbf{H}_{k o}^{S_{k}^{1}}\right),\left|S_{k}\right| \leq n\right\}, \quad \forall i \neq 1
\end{aligned}
$$

where $\tau \in[0,1]$ is a constant that we use to compare the interference caused by each SU compared to the highest SU interferer at that PR. Specifically, it is the set of SUs whose interference is at least a fraction $\tau$ of the interference caused by the highest SU interferer. The set of PRs at which the $i^{t h}$ $\mathrm{SU}$ will be aligned is given as:

$$
\mathcal{A}_{i}=\left\{j \in\{1, \ldots, F\}: i \in \mathcal{S}_{j}\right\}, \quad \forall i \in\{1, \ldots, M\}
$$


Next, the IA algorithm presented in the next section is applied to the set of SUs at each PR. Note that the set of aligned users for a given PR can be different from other PRs, due to the channel conditions and the location of the users and base stations, as well as the number of SUs to be aligned at each PR. This approach allows the PR to adapt to different conditions. For example, when the number of dominant SU interferers is low, it can allocate its resources mainly for its own users, achieving high data rates by multiplexing. Also, when the number of high interferers increases, it can devote a necessary amount of its resources to align these SUs and to prevent signal degradation for its own users. It is important to note that the choice of $\tau$ as well as the maximum number of aligned users $n$ depend on the available resources, feasibility requirements and system conditions. In particular, $\tau$ is a parameter that allows us to control the number of elements of each set. A larger $\tau$ results in fewer number of users to be aligned at a given destination. As a result, when IA is not feasible, we can increase $\tau$ to reduce the number of users to be aligned at each receiver. The number of SUs aligned at each PR cannot exceed $n$, which is introduced to control the maximum number of elements of each set. These are essentially empirical parameters to help the system designer to control the user selection process. They can be determined at a central unit and be communicated to the PRs/femtocells via the backhaul.

\section{Cross-Tier Interference Alignment}

\section{A. Formulation}

In order to align the dominant SU interferers, we define the interference subspaces at each PR such that the received signals from the selected SUs at each PR will span the subspace specific to that PR. For this purpose, we define matrices $\mathbf{V}_{1}, \mathbf{V}_{2}, \ldots, \mathbf{V}_{F}$ such that the columns of these matrices define the basis for the subspaces for the aligned interference at each receiver. That is, each column of $\mathbf{H}_{k o}^{j} \mathbf{W}_{o j}$ can be written as a linear combination of the columns of $\mathbf{V}_{k}$, $\forall j \in \mathcal{S}_{k}$, and $\forall k \in\{1, \ldots, F\}$. The IA condition requires that the received signals from the SU set defined for each PR span the same subspace, which is given as:

$$
\mathbf{H}_{k o}^{j} \mathbf{W}_{o j} \prec \mathbf{V}_{k}, \quad \forall j \in \mathcal{S}_{k}, \quad \forall k \in\{1, \ldots, F\}
$$

where $\mathbf{X} \prec \mathbf{Y}$ denotes that the column space of $\mathbf{Y}$ spans that of $\mathbf{X}$. We denote the $i^{t h}$ column of $\mathbf{V}_{k}$ by $\mathbf{v}_{k}^{i}$, or equivalently $\mathbf{V}_{k}=\left[\mathbf{v}_{k}^{1} \mathbf{v}_{k}^{2} \ldots \mathbf{v}_{k}^{d}\right]$, and the $i^{t h}$ column of $\mathbf{W}_{o j}$ as $\mathbf{w}_{o j}^{i}$, i.e., $\mathbf{W}_{o j}=\left[\mathbf{w}_{o j}^{1} \mathbf{w}_{o j}^{2} \ldots \mathbf{w}_{o j}^{d}\right]$. Then the conditions for IA [21] at PRs $k=1, \ldots F$ are:

$\mathbf{H}_{k o}^{j} \mathbf{w}_{o j}^{i}=\alpha_{k j}^{i} \mathbf{v}_{k}^{1}+\beta_{k j}^{i} \mathbf{v}_{k}^{2}+\ldots+\theta_{k j}^{i} \mathbf{v}_{k}^{d}, \quad \forall j \in \mathcal{S}_{k}, \quad i=1, \ldots, d$

where $\alpha_{k j}^{i}$ is a constant and the given equations require that all the SUs that are in the "interference set" of a PR span the same column space. In other words, received signals from selected SUs are represented by a linear combination of the subspace basis vectors, scaled by different coefficients. The conditions in (5) can be represented as follows:

$$
\widetilde{\mathbf{H}}_{k j} \mathbf{w}_{o j}=\widetilde{\mathbf{A}}_{k j} \mathbf{v}_{k}, \quad \forall j \in \mathcal{S}_{k}
$$

where $\mathbf{w}_{o j}=\left[\begin{array}{llll}\left(\mathbf{w}_{o j}^{1}\right)^{T} & \left(\mathbf{w}_{o j}^{2}\right)^{T} & \ldots & \left(\mathbf{w}_{o j}^{d}\right)^{T}\end{array}\right]^{T} \forall j \in \mathcal{S}_{k}$, $\mathbf{v}_{k}=\left[\begin{array}{llll}\left(\mathbf{v}_{k}^{1}\right)^{T} & \left(\mathbf{v}_{k}^{2}\right)^{T} & \ldots & \left(\mathbf{v}_{k}^{d}\right)^{T}\end{array}\right]^{T} . \widetilde{\mathbf{H}}_{k j}$ is a block diagonal matrix with $d$ blocks of $\mathbf{H}_{k o}^{j}$ :

$$
\widetilde{\mathbf{H}}_{k j}=\left[\begin{array}{cccc}
\mathbf{H}_{k o}^{j} & \mathbf{0} & \ldots & \mathbf{0} \\
\mathbf{0} & \mathbf{H}_{k o}^{j} & \ldots & \mathbf{0} \\
\vdots & & \ddots & \vdots \\
\mathbf{0} & \ldots & \mathbf{0} & \mathbf{H}_{k o}^{j}
\end{array}\right], \quad \forall j \in \mathcal{S}_{k}
$$

The coefficient matrices $\widetilde{\mathbf{A}}_{k j}$ for $\forall j \in \mathcal{S}_{k}$ are:

$$
\widetilde{\mathbf{A}}_{k j}=\left[\begin{array}{cccc}
\alpha_{k j}^{1} & \beta_{k j}^{1} & \ldots & \theta_{k j}^{1} \\
\alpha_{k j}^{2} & \beta_{k j}^{2} & \ldots & \theta_{k j}^{2} \\
\vdots & & \ddots & \vdots \\
\alpha_{k j}^{d} & \beta_{k j}^{d} & \ldots & \theta_{k j}^{d}
\end{array}\right] \otimes \mathbf{I}_{N_{f} \times N_{f}}
$$

where $\mathbf{I}_{N_{f} \times N_{f}}$ denotes the $\left(N_{f} \times N_{f}\right)$ identity matrix, and

$$
\mathbf{A}_{k j}=\left[\begin{array}{cccc}
\alpha_{k j}^{1} & \beta_{k j}^{1} & \ldots & \theta_{k j}^{1} \\
\alpha_{k j}^{2} & \beta_{k j}^{2} & \ldots & \theta_{k j}^{2} \\
\vdots & & \ddots & \vdots \\
\alpha_{k j}^{d} & \beta_{k j}^{d} & \ldots & \theta_{k j}^{d}
\end{array}\right] .
$$

When we follow this procedure for each receiver, the necessary conditions for IA at $F$ PRs can be represented as:

$$
\widetilde{\mathbf{H}}_{k j} \mathbf{w}_{o j}=\widetilde{\mathbf{A}}_{k j} \mathbf{v}_{k}, \quad \forall j \in \mathcal{S}_{k}, \quad \forall k \in\{1, \ldots, F\}
$$

\section{B. Algorithm}

The proposed distributed algorithm is as follows:

1) Initialize the matrices $\mathbf{V}_{1}, \mathbf{V}_{2}, \ldots, \mathbf{V}_{F}$ and $\mathbf{A}_{k j}$ for $k=$ $1, \ldots, F$ and $j=1, \ldots, M$, with

elements drawn independently from the standard normal distribution $\mathcal{N}(0,1)$.

2) Determine the precoding vectors $\mathbf{w}_{o 1}, \mathbf{w}_{o 2}, \ldots, \mathbf{w}_{o M}$ as:

$$
\begin{array}{cl}
\mathbf{w}_{o j}=\arg \min _{\mathbf{w}_{o j}} & \sum_{k \in \mathcal{A}_{j}}\left\|\widetilde{\mathbf{H}}_{k j} \mathbf{w}_{o j}-\widetilde{\mathbf{A}}_{k j} \mathbf{v}_{k}\right\|^{2} \\
\text { s.t. } & \operatorname{tr}\left(\left(\mathbf{w}_{o j}\right)^{H} \mathbf{w}_{o j}\right)=1
\end{array}
$$

where the constraint guarantees the transmit power of each $\mathrm{SU}$ is $\operatorname{tr}\left(\left(\sqrt{p_{o j}} \mathbf{w}_{o j}\right)^{H} \sqrt{p_{o j}} \mathbf{w}_{o j}\right)=p_{o j}$.

3) Construct the precoding matrices $\mathbf{W}_{o 1}, \mathbf{W}_{o 2}, \ldots, \mathbf{W}_{o M}$ using the precoding vectors from Step 2).

4) Fix the precoding matrices and determine the vectors $\mathbf{v}_{1}, \mathbf{v}_{2}, \ldots, \mathbf{v}_{F}$ as follows:

$$
\mathbf{v}_{k}=\arg \min _{\mathbf{v}_{k}} \sum_{j \in \mathcal{S}_{k}}\left\|\widetilde{\mathbf{H}}_{k j} \mathbf{w}_{o j}-\widetilde{\mathbf{A}}_{k j} \mathbf{v}_{k}\right\|^{2}
$$

5) Determine the coefficients $\mathbf{A}_{k j}$ for $k=1, \ldots, F$ and $j=$ $1, \ldots, M$ according to the following procedure. For a given $\mathbf{H}_{k o}^{j}, \mathbf{W}_{o j}$ and $\mathbf{V}_{k}$, construct the following equation:

$$
\left(\mathbf{H}_{k o}^{j} \mathbf{w}_{o j}^{k}\right)^{T}=\underbrace{\left[\begin{array}{llll}
\alpha_{k j}^{i} & \beta_{k j}^{i} & \ldots & \theta_{k j}^{i}
\end{array}\right]}_{\left(\mathbf{a}_{k j}^{i}\right)^{T}}\left(\mathbf{V}_{k}\right)^{T}
$$

Then $\mathbf{a}_{k j}^{i}=\mathbf{V}_{k}^{\dagger} \mathbf{H}_{k o}^{j} \mathbf{w}_{o j}^{i}$, and $\mathbf{A}_{k j}^{T}=\left[\begin{array}{llll}\mathbf{a}_{k j}^{1} & \mathbf{a}_{k j}^{2} & \ldots & \mathbf{a}_{k j}^{d}\end{array}\right]$ where $\mathbf{V}_{k}^{\dagger}=\left(\mathbf{V}_{k}^{H} \mathbf{V}_{k}\right)^{-1} \mathbf{V}_{k}^{H}$ denotes the pseudo-inverse of the matrix $\mathbf{V}_{k}$. 
6) Iterate from Step 2) to Step 5) until convergence.

This algorithm is distributed in the sense that each SU needs to know the channel gains from itself to the receivers it is interfering with, so that it can apply the algorithm and determine its own precoding vector. Each $\mathrm{SU}$ also needs to know $\widetilde{\mathbf{A}}_{k j} \mathbf{v}_{k}, \forall k \in \mathcal{A}_{j}$ (for the $j^{t h} \mathrm{SU}$ ).

\section{Details of Steps 2) and 4)}

Problem (11) in Step 2) can be turned into a quadratically constrained quadratic problem (QCQP) by relaxing the equality constraint into an inequality constraint:

$$
\begin{array}{cl}
\mathbf{w}_{o j}=\arg \min _{\mathbf{w}_{o j}} & \sum_{k \in \mathcal{A}_{j}}\left\|\widetilde{\mathbf{H}}_{k j} \mathbf{w}_{o j}-\widetilde{\mathbf{A}}_{k j} \mathbf{v}_{k}\right\|^{2} \\
\text { s.t. } & \operatorname{tr}\left(\left(\mathbf{w}_{o j}\right)^{H} \mathbf{w}_{o j}\right) \leq 1
\end{array}
$$

Then the KKT conditions for (14) can be formulated as:

Stationarity: $\sum_{k \in \mathcal{A}_{j}} \widetilde{\mathbf{H}}_{k j} \mathbf{w}_{o j}^{*}+\lambda_{j} \mathbf{w}_{o j}^{*}-\sum_{k \in \mathcal{A}_{j}} \widetilde{\mathbf{H}}_{k j}^{H} \widetilde{\mathbf{A}}_{k j} \mathbf{v}_{k}=0$

Complementary Slackness: $\quad \lambda_{j}\left(\left(\mathbf{w}_{o j}^{*}\right)^{H} \mathbf{w}_{o j}^{*}-1\right)=0$

Dual feasibility: $\quad \lambda_{j} \geq 0$

Primary feasibility: $\left(\mathbf{w}_{o j}^{*}\right)^{H} \mathbf{w}_{o j}^{*} \leq 1$

where $\mathbf{w}_{o j}^{*}$ denotes the optimal $\mathbf{w}_{o j}$. The optimal $\mathbf{w}_{o j}$ for $j=1, \ldots, M, \mathbf{w}_{o j}^{*}$, are those that satisfy the KKT conditions (15.a)-(15.d) which can be expressed as:

$$
\mathbf{w}_{o j}^{*}=\left(\sum_{k \in \mathcal{A}_{j}}\left(\widetilde{\mathbf{H}}_{k j}\right)^{H} \widetilde{\mathbf{H}}_{k j}+\lambda_{j} \mathbf{I}\right)^{-1} \sum_{k \in \mathcal{A}_{j}} \widetilde{\mathbf{H}}_{k j}^{H} \widetilde{\mathbf{A}}_{k j} \mathbf{v}_{k}
$$

where $\lambda_{k}$ is calculated such that $\operatorname{tr}\left(\left(\mathbf{w}_{o k}\right)^{H} \mathbf{w}_{o k}\right)=1$. The procedure for obtaining the optimal values using the KKT conditions for convex QCQPs can be found in [22]. The optimality condition for the unconstrained problem (12) is:

$$
\sum_{j \in \mathcal{S}_{k}} \widetilde{\mathbf{A}}_{k j}^{H} \widetilde{\mathbf{A}}_{k j} \mathbf{v}_{k}^{*}-\sum_{j \in \mathcal{S}_{k}} \widetilde{\mathbf{A}}_{k j}^{H} \widetilde{\mathbf{H}}_{k j} \mathbf{w}_{o j}=0
$$

where $\mathbf{v}_{k}^{*}$ is the optimal $\mathbf{v}_{k}$. Using this condition, we obtain the optimal $\mathbf{v}_{k}$ for $k=1, \ldots, F$ as:

$$
\mathbf{v}_{k}^{*}=\left(\sum_{j \in \mathcal{S}_{k}} \widetilde{\mathbf{A}}_{k j}^{H} \widetilde{\mathbf{A}}_{k j}\right)^{-1}\left(\sum_{j \in \mathcal{S}_{k}} \widetilde{\mathbf{A}}_{k j}^{H} \widetilde{\mathbf{H}}_{k j} \mathbf{w}_{o j}\right)
$$

Using (18), we can determine the interference subspace matrices $\mathbf{V}_{1}, \ldots, \mathbf{V}_{F}$.

\section{Convergence Analysis}

In this section, we provide the convergence analysis for the algorithm proposed in Section IV. We define the total leaked interference from all SUs and PRs as:

$$
\begin{aligned}
Q & =\sum_{k=1}^{F} \sum_{j \in \mathcal{S}_{k}}\left\|\widetilde{\mathbf{H}}_{k j} \mathbf{w}_{o j}-\widetilde{\mathbf{A}}_{k j} \mathbf{v}_{k}\right\|^{2} \\
& =\sum_{j=1}^{M} \sum_{k \in \mathcal{A}_{j}}\left\|\widetilde{\mathbf{H}}_{k j} \mathbf{w}_{o j}-\widetilde{\mathbf{A}}_{k j} \mathbf{v}_{k}\right\|^{2}
\end{aligned}
$$

When $\mathbf{v}_{1}, \ldots, \mathbf{v}_{F}$ are fixed, $\mathbf{w}_{o 1}, \ldots, \mathbf{w}_{o M}$ are determined according to (11), which decreases the value of $Q$ given by
(20). Similarly, when $\mathbf{w}_{o 1}, \ldots, \mathbf{w}_{o M}$ are fixed, we determine $\mathbf{v}_{1}, \ldots, \mathbf{v}_{F}$ using (12), from which we see that the value of $Q$ given by (19) is also decreased. Thus $Q$ is decreased after each iteration, and since $Q$ is bounded below by zero, the algorithm converges. Although due to the non convex nature of the problem this algorithm is guaranteed to converge to a local optimum only, our numerical results indicate that the leakage interference can converge to values very close to zero which is a lower bound to the global optimum.

\section{Precoder and Decoder Design for the Primary USERS}

The precoders and decoders for the primary/femtocell users are constructed using the MMSE criterion. We first define the estimated signal for the $j^{t h}$ user of the $k^{t h}$ femtocell as follows:

$$
\begin{aligned}
\hat{\mathbf{s}}_{k j}= & \sum_{i=1}^{U_{k}} \sqrt{p_{k i}}\left(\mathbf{G}_{k j}\right)^{H} \mathbf{H}_{k k}^{i} \mathbf{W}_{k i} \mathbf{s}_{k i} \\
& +\sum_{\substack{f=1 \\
f \neq k}}^{F} \sum_{u=1}^{U_{f}} \sqrt{p_{f u}}\left(\mathbf{G}_{k j}\right)^{H} \mathbf{H}_{k f}^{u} \mathbf{W}_{f u} \mathbf{s}_{f u} \\
& +\sum_{m=1}^{M} \sqrt{p_{o m}}\left(\mathbf{G}_{k j}\right)^{H} \mathbf{H}_{k o}^{m} \mathbf{W}_{o m} \mathbf{s}_{o m}+\left(\mathbf{G}_{k j}\right)^{H} \mathbf{n}_{k}
\end{aligned}
$$

where $\mathbf{G}_{k j}$ is the decoding matrix of the $j^{t h}$ user of the $k^{t h}$ femtocell. Since the interference caused by other femtocells is very small compared to the intracell interference, for simplicity we will regard intercell femtocell interference as noise, which is given as:

$$
\tilde{\mathbf{n}}_{k}=\sum_{\substack{f=1 \\ f \neq k}}^{F} \sum_{u=1}^{U_{f}} \sqrt{p_{f u}} \mathbf{H}_{k f}^{u} \mathbf{W}_{f u} \mathbf{s}_{f u}+\mathbf{n}_{k}
$$

Then the minimum sum MSE problem at the $k^{\text {th }}$ primary receiver/femtocell base station is:

$$
\begin{array}{ll}
\underset{\substack{\mathbf{W}_{k 1}, \ldots, \mathbf{W}_{k U_{k}} \\
\mathbf{G}_{k 1}, \ldots, \mathbf{G}_{k U_{k}}}}{\operatorname{minimize}} & \sum_{j=1}^{U_{k}} E\left\{\left\|\hat{\mathbf{s}}_{k j}-\mathbf{s}_{k j}\right\|^{2}\right\} \\
\text { subject to } & \operatorname{tr}\left(\left(\mathbf{W}_{k j}\right)^{H} \mathbf{W}_{k j}\right)=1 \quad j=1, \ldots, U_{k}
\end{array}
$$

where the equality constraint is used to guarantee that the transmit power of the femtocell users satisfies $\operatorname{tr}\left(\left(\sqrt{p_{k j}} \mathbf{W}_{k j}\right)^{H} \sqrt{p_{k j}} \mathbf{W}_{k j}\right)=p_{k j}$. We denote the covariance matrix of $\tilde{\mathbf{n}}_{k}$ as $E\left\{\tilde{\mathbf{n}}_{k}\left(\tilde{\mathbf{n}}_{k}\right)^{H}\right\}=\mathbf{R}_{\tilde{n}}$. The optimization problem in (23) can be relaxed into a QCQP by replacing the equality constraint with an inequality, as in (14). The resulting problem is convex in $\mathbf{W}_{k j}$ if all other $\mathbf{G}_{k j}$ are fixed, and convex in $\mathbf{G}_{k j}$ if all other $\mathbf{W}_{k j}$ are fixed. Therefore, we can use an iterative algorithm such as alternating minimization by fixing the decoding matrices first to determine the precoding matrices, and then fixing the precoding matrices to determine the decoding matrices. An iterative minimization procedure is defined in [23] to determine the coding vectors in a MIMO system with unit norm transmit precoders. The solution for the optimal precoding and decoding matrices of the primary/femtocell 
users is then determined by using the Lagrangian and the KKT conditions for the sum MMSE problem. The resulting precoders and decoders are:

$$
\begin{aligned}
\mathbf{G}_{k j} & =\left(\sum_{i=1}^{U_{k}} p_{k i} \mathbf{H}_{k k}^{i} \mathbf{W}_{k i}\left(\mathbf{H}_{k k}^{i} \mathbf{W}_{k i}\right)^{H}\right. \\
+ & \left.\sum_{m=1}^{M} p_{o m} \mathbf{H}_{k o}^{m} \mathbf{W}_{o m}\left(\mathbf{H}_{k o}^{m} \mathbf{W}_{o m}\right)^{H}+\mathbf{R}_{\tilde{n}}\right)^{-1} \sqrt{p_{k j}} \mathbf{H}_{k k}^{j} \mathbf{W}_{k j} \\
\mathbf{W}_{k j}= & \left(\sum_{i=1}^{U_{k}} p_{k i}\left(\mathbf{H}_{k k}^{j}\right)^{H} \mathbf{G}_{k i}\left(\mathbf{G}_{k i}\right)^{H} \mathbf{H}_{k k}^{j}\right. \\
& \left.+\mu_{k j} \mathbf{I}\right)^{-1} \sqrt{p_{k j}}\left(\mathbf{H}_{k k}^{j}\right)^{H} \mathbf{G}_{k j}
\end{aligned}
$$

for $j=1, \ldots, U_{k}$. We determine $\mu_{k j}$ such that $\operatorname{tr}\left(\left(\mathbf{W}_{k j}\right)^{H} \mathbf{W}_{k j}\right)=1$.

\section{DISCUSSION}

It is at this point useful to discuss the reasons for being able to use the distributed approach in Section V. If we did not specify the subspaces for each PR, and still wanted to use IA, then the SUs would have to share information with other SUs (such as channel and coefficient information), which is not preferred due to the excessive load on mobile users and privacy issues. If we did not want to share information between users, we would have to use a centralized algorithm as in [17], but since in the present scheme we are considering the whole femtocell network instead of a group of PRs, using a centralized method would require sending the channel information and the information about the IA sets for each PR before each transmission to a centralized processor, and the centralized processor would solve the IA problem with excessive amounts of data, and send back the determined precoders to the SUs, over the SR-SUs link. Instead, we have essentially divided this single problem into multiple problems that can be solved at each PR locally, in parallel with other PRs which saves from this overhead. In the proposed scheme, each PR needs to share channel and coefficient information only with the SUs in its IA set $\mathcal{S}_{k}$ to create its IA subspace. The SUs only use the information about the subspaces of the PRs in their IA set $\mathcal{A}_{j}$ and their channels to those PRs. The sole constraint for the IA problem is the transmit power constraint, and no other assumptions are made on the precoders/subspaces, which renders the problem easy to relax into different types, such as an SDP problem, and add additional constraints such as minimum SINR requirements, which can be employed for improving the QoS of SUs [17], if so desired.

\section{FeAsibility of SElective Interference AlignMent}

In this section we will discuss the feasibility of the proposed method and the conditions for perfect IA. We will first determine the conditions for which the system is proper, i.e., the number of variables to be determined is greater than or equal to the number of equations in the system. In order to find the number of equations, we reformulate the IA conditions in (5) as the following:

$$
\mathbf{H}_{k o}^{j} \mathbf{W}_{o j}=\mathbf{V}_{k} \widetilde{\mathbf{A}}_{k j}^{H}, \quad \forall j \in \mathcal{S}_{k}, \quad k \in\{1, \ldots, F\}
$$

Then the number of equations $N_{e q}$ can be obtained as

$$
N_{e q}=\sum_{f=1}^{F}\left|\mathcal{S}_{f}\right| N_{f} d=d \sum_{f=1}^{F}\left|\mathcal{S}_{f}\right| N_{f}=d \sum_{j=1}^{M} \sum_{f \in \mathcal{A}_{j}} N_{f}
$$

The number of variables $N_{v a r}$ to be determined in the proposed system is obtained by eliminating the superfluous variables for IA [25]. The fact that the basis representation for a subspace is not unique can be used to achieve the minimum number of variables to be determined for each subspace matrix $\mathbf{V}_{k}$. In particular, post-multiplying $\mathbf{V}_{k}$ with any full rank $d \times d$ matrix $\mathbf{P}$ will not change the subspace spanned by $\mathbf{V}_{k}$ :

$$
\begin{aligned}
\operatorname{span}\left(\mathbf{V}_{k}\right) & =\left\{\mathbf{y}: \exists \mathbf{x} \in \mathbb{C}^{d \times 1}, \mathbf{y}=\mathbf{V}_{k} \mathbf{x}\right\} \\
& =\left\{\mathbf{y}: \exists \mathbf{x} \in \mathbb{C}^{d \times 1}, \mathbf{y}=\mathbf{V}_{k} \mathbf{P}_{k}^{-1} \mathbf{P}_{k} \mathbf{x}\right\} \\
& =\operatorname{span}\left(\mathbf{V}_{k} \mathbf{P}_{k}^{-1}\right)
\end{aligned}
$$

We can select the matrix $\mathbf{P}_{k}$ obtained by removing the last $N_{f}-d$ rows of $\mathbf{V}_{k}$. Then the modified subspace matrix $\mathbf{V}_{k} \mathbf{P}_{k}^{-1}=\overline{\mathbf{V}}_{k}$ has the following form:

$$
\overline{\mathbf{V}}_{k}=\left[\begin{array}{ccccc} 
& & \mathbf{I}_{(d \times d)} & & \\
\overline{\mathbf{v}}_{k}^{1} & \overline{\mathbf{v}}_{k}^{2} & \overline{\mathbf{v}}_{k}^{3} & \ldots & \overline{\mathbf{v}}_{k}^{d}
\end{array}\right]
$$

where $\overline{\mathbf{v}}_{k}^{1}, \ldots, \overline{\mathbf{v}}_{k}^{d}$ are $\left(N_{f}-d\right) \times 1$ vectors. Using a similar argument as in [25], we can see that this basis representation has the minimum number of variables to be determined. Therefore the minimum number of variables to be determined for the received interference subspace at the $k^{t h} \mathrm{PR}$ is $\left(N_{f}-d\right) d$. Following the same procedure, we can form the modified transmit subspace with the minimum number of variables for each SU precoding matrix $\mathbf{W}_{o j}, \forall j \in\{1, \ldots, M\}$. Thus the number of variables to be determined for each SU precoding matrix $\mathbf{W}_{o j}$ is $\left(N_{t}-d\right) \times d$. Accordingly, the total number of variables to be determined in the system is:

$$
N_{v a r}=M\left(N_{t}-d\right) d+\sum_{f=1}^{F}\left(N_{f}-d\right) d
$$

Theorem 1: The selective femtocell network with $F$ cognitive femtocells and $M$ SU/MUs with $N_{t}$ receive antennas at each SU/MU is proper if and only if

$$
\begin{aligned}
& N_{v a r} \geq N_{e q} \\
& \Rightarrow \sum_{f=1}^{F}\left(N_{f}-d\right) d+M\left(N_{t}-d\right) d-\sum_{f=1}^{F} N_{f}\left|\mathcal{S}_{f}\right| d \geq 0
\end{aligned}
$$

Proof: A proper system is characterized as a system such that for all subsets of equations, the number of variables is at least as large as the number of equations in that subset [25]. It can be seen that each equation involves the same number of variables for our proposed system. Therefore any deficiency in the number of variables will show up when the total number of variables is compared to the total number of equations[25]. We now state the condition for the solvability of the femtocell IA problem: 
Theorem 2: The selective IA problem for a proper cognitive femtocell network when a single beam is transmitted from each mobile user, i.e., $d=1$, is solvable almost surely when $N_{\text {var }} \geq N_{\text {eq }}$.

Proof: The equations of the femtocell IA system have independent random coefficients due to the random nature of the channel distribution. Therefore, applying Bernshteim's Theorem results in a nonnegative number of common solutions, which is equal to the mixed volume of Newton polytopes.

Algebraic geometry details for the Newton's polytope and Bernshtein's Theorem can be found in [25], [26]. The fact that the coefficients of the system equations are not independent when multiple beams are transmitted prevents Bernshtein's Theorem from being applied to determine the solvability of multi-beam systems. A recent work on feasibility of IA [27] considers the symmetric square case in which all the transmitters and receivers have the same number of antennas. This is different from the wireless femtocell networks where mobile users and base stations usually employ a different number of antennas. Similarly, algebraic tools to find similar bounds on the tuple of DoF that are achievable through linear IA have been considered in [28]. For symmetric systems where the number of transmit and receive antennas is divisible by the number of streams, the bound is tight and can be achieved through IA.

\section{Downlink InTERfEREnCE ALIGNMENT}

In this section, we consider the downlink of the two-tier network and cross-tier IA. In the downlink, a FBS transmitting to a FU, a close by MU that is far from the MBS may be severely affected by the FBS signal. Multiple FBSs transmitting in the close neighborhood of the MU can exacerbate this effect and end up disconnecting the macrocell communication link. In this scenario, a reasonable action could be to utilize IA for the downlink to manage the interference at the MUs caused by the FBSs, while sacrificing the minimum number of dimensions. For this purpose, at each MU, we can align the received signals from the set of FBSs (the cognitive transmitters) that are causing high interference to that $\mathrm{MU}$, and then iteratively determine the precoders of the FBSs and the interference subspaces defined at the MUs. This is of course similar to what we have done in the uplink. The primary transmitters (FBS) cooperate with the secondary receivers in the downlink to have their received interference be aligned at a lower dimensional subspace.

In this case, signal received at the $k^{\text {th }}$ macrocell user is:

$$
\mathbf{y}_{k}=\underbrace{\sqrt{p_{o} \mathbf{H}_{k o} \mathbf{W}_{o} \mathbf{s}_{o}}}_{\begin{array}{c}
\text { signal received from } \\
\text { the MBS }
\end{array}}+\underbrace{\sum_{f=1, f \neq k}^{F} \sqrt{p_{f}} \mathbf{H}_{k f} \mathbf{W}_{f} \mathbf{s}_{f}}_{\begin{array}{c}
\text { interference received from } \\
\text { the FBSs }
\end{array}}+\mathbf{n}_{k}
$$

where $\mathbf{n}_{k}$ is the Gaussian noise vector, $\mathbf{H}_{k o}$ denotes the channel from the MBS to the $k^{t h} \mathrm{MU}, \mathbf{H}_{k f}$ is the channel from the $f^{t h}$ FBS to the $k^{\text {th }} \mathrm{MU}$, and $\sqrt{p_{o}}$ and $\sqrt{p_{f}}$ are the transmit powers of the MBS and the $f^{t h}$ FBS, respectively. $\mathbf{W}_{o}=\left[\mathbf{W}_{o 1}, \mathbf{W}_{o 2}, \ldots, \mathbf{W}_{o M}\right]$ is the precoder matrix of the MBS serving macrocell users $k=1, \ldots, M$ with $\mathbf{W}_{o k}$ as the precoder for MU $k . \mathbf{W}_{f}$ represents the precoder of the $f^{t h}$ FBS. The vector $\mathbf{s}_{o}=\left[\mathbf{s}_{o 1}^{T}, \ldots, \mathbf{s}_{o M}^{T}\right]^{T}$ holds the message signals for all the MUs, in which $\mathbf{s}_{o k}$ is the message vector intended for the $k^{t h}$ MU. We use $\mathbf{s}_{f}$ for the message signal of the FBS. Precoders of the base stations satisfy $\operatorname{tr}\left(\mathbf{W}_{o}^{H} \mathbf{W}_{o}\right)=\operatorname{tr}\left(\mathbf{W}_{f}^{H} \mathbf{W}_{f}\right)=1, \forall k \in\{1, \ldots, F\}$.

Define the set of dominant FBS interferers at the $k^{\text {th }}$ MU as $\mathcal{S}_{k}=\left\{\mathcal{S}_{k}^{1}, \mathcal{S}_{k}^{2}, \ldots, \mathcal{S}_{k}^{\left|S_{k}\right|}\right\}$ where

$$
\begin{aligned}
\mathcal{S}_{k}^{1}= & \underset{j \in\{1, \ldots, F\}}{\arg \max } \operatorname{tr}\left(\left(\mathbf{H}_{k j}\right)^{H} \mathbf{H}_{k j}\right) \\
\mathcal{S}_{k}^{i}= & \left\{j \in\{1, \ldots, F\}: \operatorname{tr}\left(\left(\mathbf{H}_{k j}\right)^{H} \mathbf{H}_{k j}\right) \geq\right. \\
& \left.\tau \operatorname{tr}\left(\left(\mathbf{H}_{k S_{k}^{1}}\right)^{H} \mathbf{H}_{k S_{k}^{1}}\right),\left|S_{k}\right| \leq n\right\}, \quad \forall i \neq 1
\end{aligned}
$$

and $\tau \in[0,1]$ is a constant that we use to compare the interference caused by each MU compared to the highest MU interferer at that FBS. Specifically, it is the set of MUs whose interference is at least a fraction $\tau$ of the interference caused by the highest MU interferer. The set of FBSs at which the $i^{t h}$ MU will be aligned is given as $\mathcal{A}_{i}=\{j \in\{1, \ldots, M\}: i \in$ $\left.\mathcal{S}_{j}\right\}, \quad \forall i \in\{1, \ldots, F\}$. Defining matrices $\mathbf{V}_{1}, \mathbf{V}_{2}, \ldots, \mathbf{V}_{M}$ such that the columns of these matrices will define a basis for the interference aligned at each MU, we form the IA conditions at the MUs as follows:

$$
\mathbf{H}_{m j} \mathbf{W}_{j} \prec \mathbf{V}_{m}, \quad \forall j \in \mathcal{S}_{m}, \quad \forall m \in\{1, \ldots, M\}
$$

For convenience, we assume the same number of users at each femtocell, i.e., $U_{1}=U_{2}=\ldots=U$. We then reformulate the IA conditions at MU $k=1, \ldots, M$ using the interference subspace matrix $\mathbf{V}_{k}=\left[\mathbf{v}_{k}^{1} \mathbf{v}_{k}^{2} \ldots \mathbf{v}_{k}^{U}\right]$ and the precoders $\mathbf{W}_{o}=\left[\mathbf{w}_{o}^{1} \mathbf{w}_{o}^{2} \ldots \mathbf{w}_{o}^{M}\right]$ for the MBS and $\mathbf{W}_{j}=\left[\mathbf{w}_{j}^{1} \mathbf{w}_{j}^{2} \ldots \mathbf{w}_{j}^{U}\right]$ for the FBSs $j=1, \ldots, F$ as follows: $\mathbf{H}_{k j} \mathbf{w}_{j}^{i}=\alpha_{k j}^{i} \mathbf{v}_{k}^{1}+\beta_{k j}^{i} \mathbf{v}_{k}^{2}+\ldots+\theta_{k j}^{i} \mathbf{v}_{k}^{U}, \forall j \in \mathcal{S}_{k}, \quad i=1, \ldots, U$

where $\alpha_{k j}^{i}$ is a constant and the given equations state that all the FBSs contained in the "interference set" of a MU span the same low dimensional subspace. We then reformulate the IA conditions at $M$ MUs using the following linear equations:

$$
\widetilde{\mathbf{H}}_{k j} \mathbf{w}_{j}=\widetilde{\mathbf{A}}_{k j} \mathbf{v}_{k}, \quad \forall j \in \mathcal{S}_{k}, \quad k \in\{1, \ldots, M\}
$$

where $\mathbf{w}_{j}=\left[\begin{array}{llll}\left(\mathbf{w}_{j}^{1}\right)^{T} & \left(\mathbf{w}_{j}^{2}\right)^{T} & \ldots & \left(\mathbf{w}_{j}^{U}\right)^{T}\end{array}\right]^{T} \forall j \in \mathcal{S}_{k}$, and $\mathbf{v}_{k}=\left[\begin{array}{llll}\left(\mathbf{v}_{k}^{1}\right)^{T} & \left(\mathbf{v}_{k}^{2}\right)^{T} & \ldots & \left(\mathbf{v}_{k}^{U}\right)^{T}\end{array}\right]^{T} . \widetilde{\mathbf{H}}_{k j}, \widetilde{\mathbf{A}}_{k j}$ and $\mathbf{A}_{k j}$ are defined as in the uplink (7)-(9). In order to construct the distributed IA algorithm for the downlink, we first initialize the matrices $\mathbf{V}_{1}, \mathbf{V}_{2}, \ldots, \mathbf{V}_{M}$ and $\mathbf{A}_{k j}$ for $k=1, \ldots, M$ and $j=1, \ldots, F$. Then we determine $\mathbf{w}_{1}, \mathbf{w}_{2}, \ldots, \mathbf{w}_{F}$ from:

$$
\begin{array}{cl}
\mathbf{w}_{j}=\arg \min _{\mathbf{w}_{j}} & \sum_{k \in \mathcal{A}_{j}}\left\|\widetilde{\mathbf{H}}_{k j} \mathbf{w}_{j}-\widetilde{\mathbf{A}}_{k j} \mathbf{v}_{k}\right\|^{2} \\
\text { s.t. } & \operatorname{tr}\left(\left(\mathbf{w}_{j}\right)^{H} \mathbf{w}_{j}\right)=1
\end{array}
$$

The equality constraint is used to ensure that the femtocell base stations satisfy the transmit power condition $\operatorname{tr}\left(\left(\sqrt{p_{j}} \mathbf{w}_{j}\right)^{H} \sqrt{p_{j}} \mathbf{w}_{j}\right)=p_{j}$, for $j=1, \ldots, F$. We then construct the precoding matrices of the FBSs $\mathbf{W}_{1}, \mathbf{W}_{2}, \ldots, \mathbf{W}_{F}$ using vectors $\mathbf{w}_{1}, \mathbf{w}_{2}, \ldots, \mathbf{w}_{F}$.

The coefficients $\mathbf{A}_{k j}$ for $k=1, \ldots, M$ and $j=1, \ldots, F$ can be determined by using (13). Then $\mathbf{a}_{k j}^{i}=\mathbf{V}_{k}^{\dagger} \mathbf{H}_{k j} \mathbf{w}_{j}^{i}$, and $\mathbf{A}_{k j}^{T}=\left[\begin{array}{llll}\mathbf{a}_{k j}^{1} & \mathbf{a}_{k j}^{2} & \ldots & \mathbf{a}_{k j}^{U}\end{array}\right]$. Next we fix the precoding 


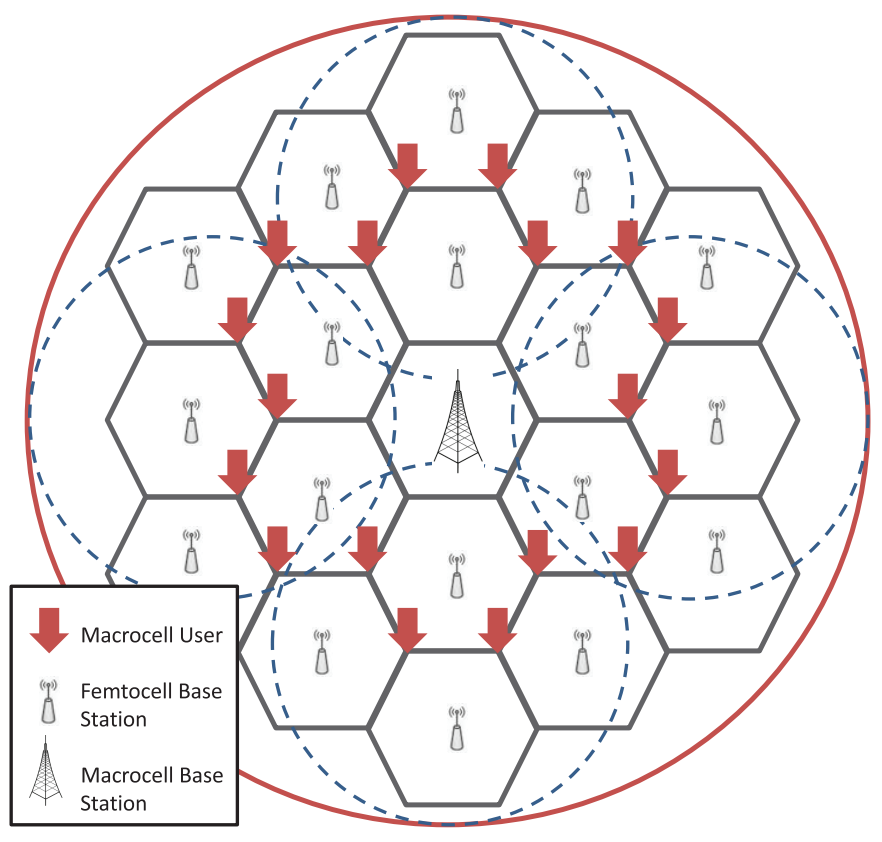

Fig. 3. Model for the dense urban femtocell network.

matrices of the FBSs and determine $\mathbf{v}_{1}, \mathbf{v}_{2}, \ldots, \mathbf{v}_{M}$ as:

$$
\mathbf{v}_{k}=\arg \min _{\mathbf{v}_{k}} \sum_{j \in \mathcal{S}_{k}}\left\|\widetilde{\mathbf{H}}_{k j} \mathbf{w}_{j}-\widetilde{\mathbf{A}}_{k j} \mathbf{v}_{k}\right\|^{2}
$$

We then iterate (37-38) until convergence. An MMSE precoder is utilized at the MBS for the $k^{\text {th }} \mathrm{MU}$ as given in (39). ${ }^{2}$

$$
\mathbf{W}_{o k}=\left(\sum_{j=1}^{M} \mathbf{H}_{j o}^{H} \mathbf{G}_{o j} \mathbf{G}_{o j}^{H} \mathbf{H}_{j o}+\lambda \mathbf{I}\right)^{-1} \mathbf{H}_{k o}^{H} \mathbf{G}_{o k}
$$

where $\mathbf{W}_{o k}$ is the precoder designed at the MBS for the $k^{t h} \mathrm{MU}$ and $\mathbf{G}_{o j}$ is the decoder at the $j^{t h} \mathrm{MU}$. Then the precoder designed at the MBS is given as $\mathbf{W}_{o}=$ $\left[\begin{array}{lll}\mathbf{W}_{o 1} & \mathbf{W}_{o 2} & \mathbf{W}_{o 3} \ldots \mathbf{W}_{o M}\end{array}\right] . \lambda$ is determined such that $\operatorname{tr}\left(\mathbf{W}_{o}^{H} \mathbf{W}_{o}\right)=P$ where $P$ is the transmit power of the MBS. Similarly, the decoder for the $k^{\text {th }} \mathrm{MU}$ is given as:

$$
\begin{aligned}
\mathbf{G}_{o k}= & \left(\sum_{j=1}^{M} \mathbf{H}_{k o} \mathbf{W}_{o j} \mathbf{W}_{o j}^{H} \mathbf{H}_{k o}^{H}+\sum_{f=1}^{F} \mathbf{H}_{k f} \mathbf{W}_{f} \mathbf{W}_{f}^{H} \mathbf{H}_{k f}^{H}\right. \\
& \left.+\sigma^{2} \mathbf{I}\right)^{-1} \mathbf{H}_{k o} \mathbf{W}_{o k}
\end{aligned}
$$

\section{Simulation Results}

Simulations are performed to compare the performance of the FUs with one MBS and multiple FBSs for two scenarios. The first scenario considers a dense urban model, with 18 FBSs distributed over a macrocell area with $155 \mathrm{~m}$ radius. The MUs are placed at the cell edges of the FBSs to analyze a worst-case cross-tier interference, see Fig. 3. Each hexagonal cell in Fig. 3 denotes a femtocell and is approximated by a circular area with a radius of $30 \mathrm{~m}$. The second scenario has a MBS with a coverage radius of $500 \mathrm{~m}$ and 14 FBSs distributed randomly, each with a coverage radius of $30 \mathrm{~m}$, this model is

\footnotetext{
${ }^{2}$ We omit the derivation of (39) due to space considerations and state that the basic idea is to follow the steps introduced in Section V.
}

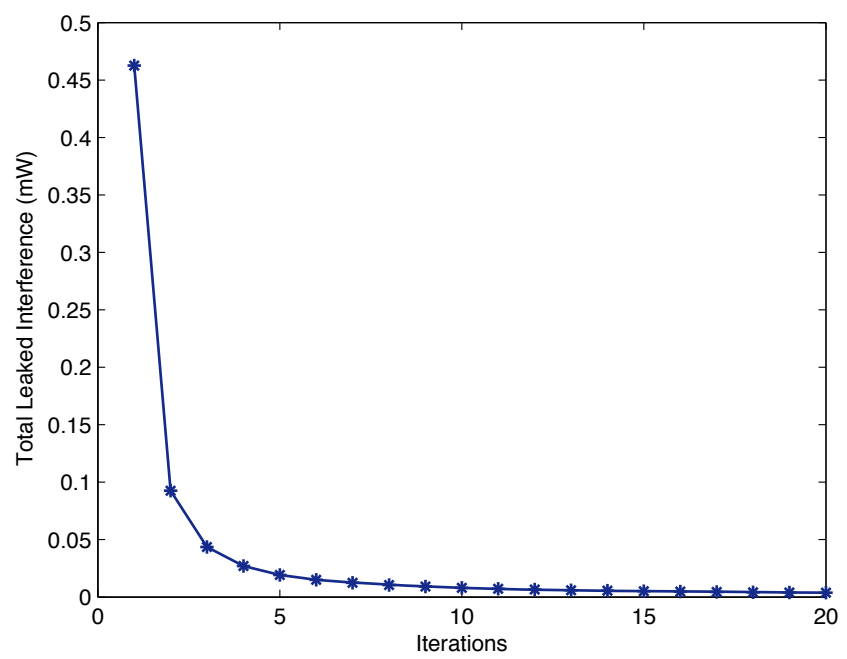

Fig. 4. Convergence of the selective IA algorithm for the uplink, with $M=18$ macrocell users.

similar to the one in Fig. 1. In both systems, each femtocell has 3 randomly distributed FUs, the mobile users have 4 transmit antennas, and the FBSs have 6 receive antennas. We use the same number of iterations across algorithms for a fair comparison. Our results are presented with precoders after convergence. ${ }^{3}$ Noise power is assumed to be $-110 \mathrm{~dB}$. We consider Rayleigh fading channels with the indoor/outdoor path loss modeled according to the ITU-R channel model [16] specifications. We assume that users in the same tier have identical transmit powers, and that macrocell users have larger transmit powers than the femtocell users in general. These power values are indicated on the plots in which we demonstrate their impact on the system performance.

Selective IA is compared with a baseline scheme without IA, in which MUs utilize MMSE precoders to improve their own performance, and FBSs use MMSE interference suppression decoders to manage the received interference, termed without IA throughout this section. We also compare it with the scheme when the macrocell coverage area is divided into smaller areas to form a femtocell group [17]. MUs and the FBSs within this group cooperate to apply IA to the received signals of the MUs at the FBSs, and the primary precoder/decoder design follows from Section V, termed IAMMSE with grouping. The radius for a femtocell group for this scheme is assumed to be $75 \mathrm{~m}$, as presented in Fig. 3 .

We begin by considering the uplink of the first scenario. The convergence of the selective IA algorithm is demonstrated in Fig. 4. Average BER performance of the PUs (FUs) with respect to the transmit powers of the MUs is shown in Fig. 5 for $M=18$. A single bit stream is transmitted from each user, i.e., $d=1$. We use Monte Carlo simulations. Average BER is calculated by dividing the sum of the bit error rates (BERs) of the FUs in all femtocells by the total number of FUs in the whole network. Individual BER of each FU is the total number of bit errors that occur after decoding at the designated FBS divided by the total number of bits transmitted

\footnotetext{
${ }^{3}$ In all our experiments we observed convergence within 20 iterations.
} 


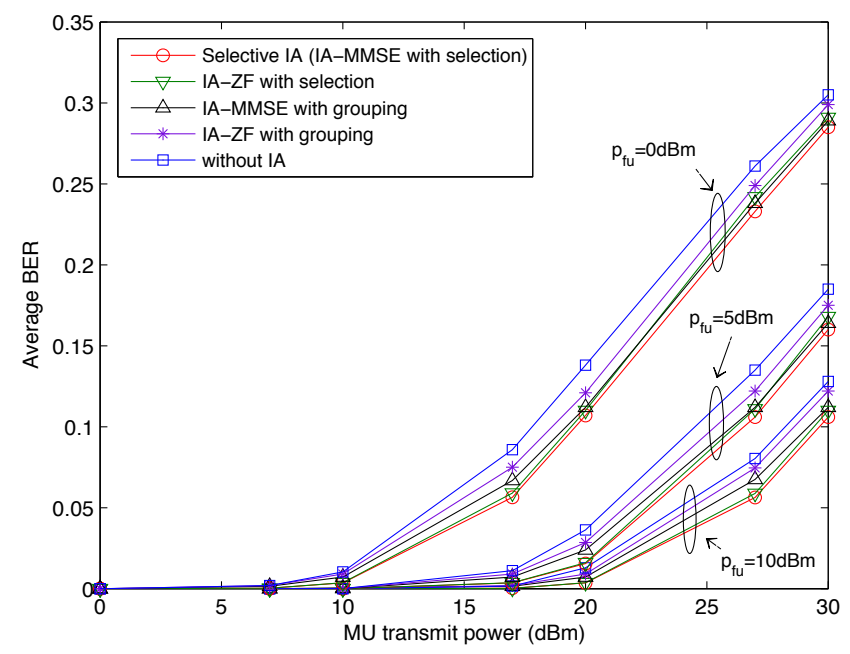

Fig. 5. Average BER of the femtocell users wrt. MU transmit power for various $\mathrm{FU}$ transmit power values.

from that user during the transmission interval. We assume that $10^{6}$ symbols are transmitted at each interval. The number of transmit antennas of the FBSs is assumed to be 6 .

For comparison purposes we also consider another scheme termed $I A-Z F$ in Fig. 5 where aligned macrocell interference is nulled at the PRs and the primary communication is restricted to the $N_{f}-d$ dimensional subspace. ${ }^{4}$ To do this, it suffices to add a constraint on primary decoder design that zero forces the received interference subspace $V_{i}$ at the designated PR:

$$
\mathbf{G}_{k j}^{H} \mathbf{V}_{k}=\mathbf{0}, \quad j=1, \ldots, U_{k}, \quad k=1, \ldots, F
$$

In doing so, we first project the primary decoders to the null space of the cross-tier secondary interference subspace and then minimize the sum MSE for the primary communication. This involves determining the interference subspace at each receiver. We compare two approaches to achieve this. The first one is to determine each interference subspace according to the cognitive user selection algorithm proposed in Section III and is termed IA-ZF with selection. On the other hand, the second one uses the femtocell grouping approach where all MUs that fall into a femtocell group apply IA, termed $I A-Z F$ with grouping. The results suggest that MMSE decoding alone achieves better performance. We believe this is due to the tiered nature of the problem, adding an additional zero forcing constraint for the received secondary interference cancellation ends up over-constraining the primary transceivers.

We present the performance when multiple bits are transmitted from each mobile user in Fig. 6 for $d=2$. Selective IA is compared with the baseline scheme without IA for different FBS antenna numbers in Fig. 7 where $N_{1}=N_{2}=\ldots=$ $N_{F}=N_{r}$. In Fig. 8, we present the performance comparison of Selective IA with IA with grouping, for various group sizes to highlight the advantage of selecting specific users to align. In this figure, FU transmit power is assumed to be $10 \mathrm{dBm}$ and the increasing index indicates increasing group size. Group 1 denotes the smallest group size with 3 FBSs in each group

\footnotetext{
${ }^{4}$ We thank the anonymous reviewer for suggesting this.
}

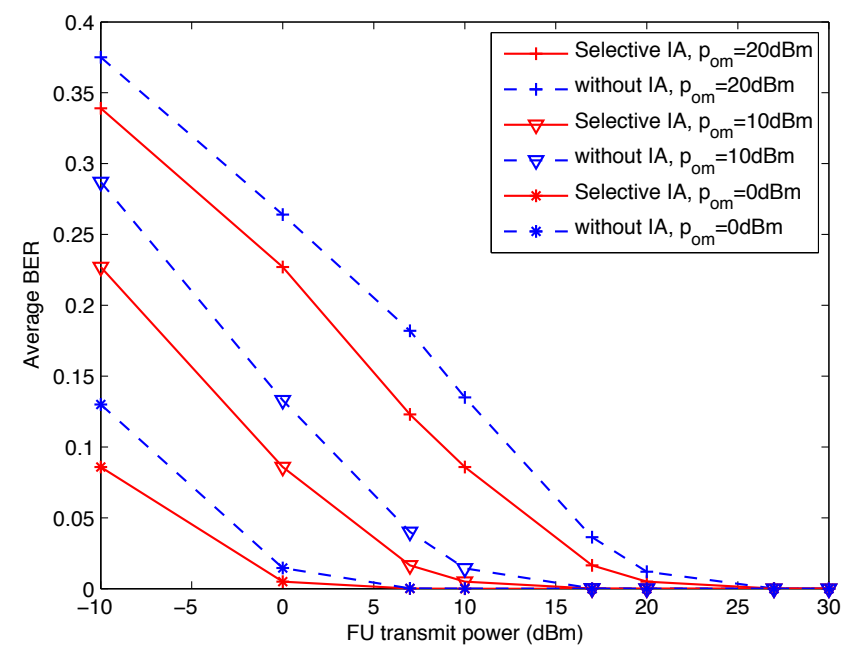

Fig. 6. Average BER of the femtocell users wrt. FU transmit power for multiple transmitted bit streams, $d=2$.

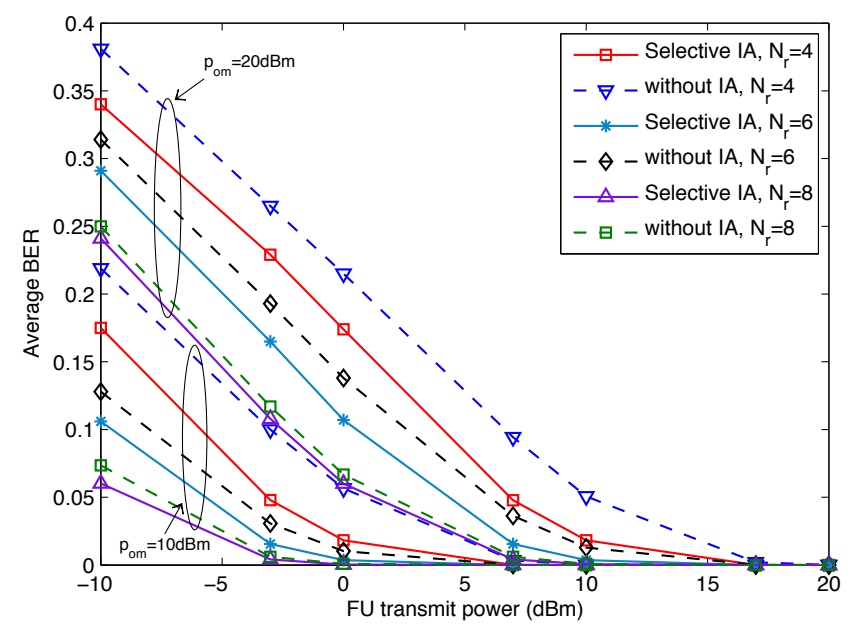

Fig. 7. Average BER of the femtocell users for different FBS antenna numbers, $N_{r}=4,6$ and 8 .

with $\left|A_{i}\right|=3$ and $\left|S_{k}\right|=3$. Group 2 is the group size used in our paper, depicted in Fig. 3, with $\left|A_{i}\right| \in\{4,5\}$ and $\left|S_{k}\right| \in\{4,5\}$. Group 3 denotes a larger size, with 6 FBSs in each group, $\left|A_{i}\right|=6$ and $\left|S_{k}\right|=6$. Group 4 is the largest group size corresponding to the case when all the FBSs apply IA. We have observed that Group 2 gives the best performance results, which is used in our simulations for comparison with selective IA. In a practical system, a fixed group size may or may not be the size that leads to the best performance. Thus, in a dynamic network, group size should to be optimized frequently to determine the membership relations.

The sum data rates for the combined macrocell-femtocell network for the selective IA, IA with grouping and without IA schemes are presented in Fig. 9. We also present the femtocell sum rate comparisons in Fig. 10 for $p_{o m}=10 \mathrm{dBm}$ $\forall m$. Table I shows the impact of parameter $\tau$ on the system performance in terms of total leaked interference, average BER of the FUs, and aggregate sum rate of the combined femtocell-macrocell network for $p_{o m}=10 \mathrm{dBm} \forall m$ and 


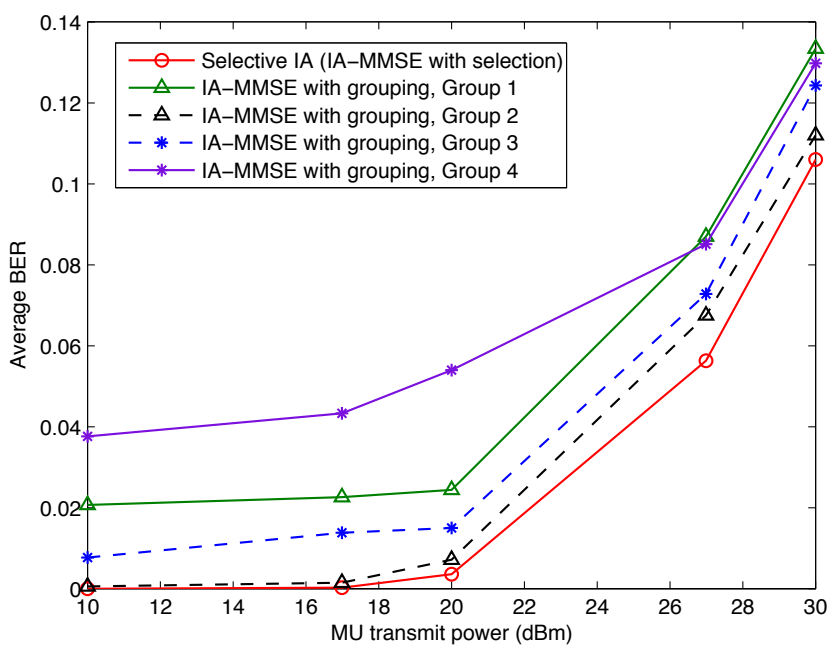

Fig. 8. Average BER of the FUs wrt MU transmit power with different group sizes for IA with user grouping.

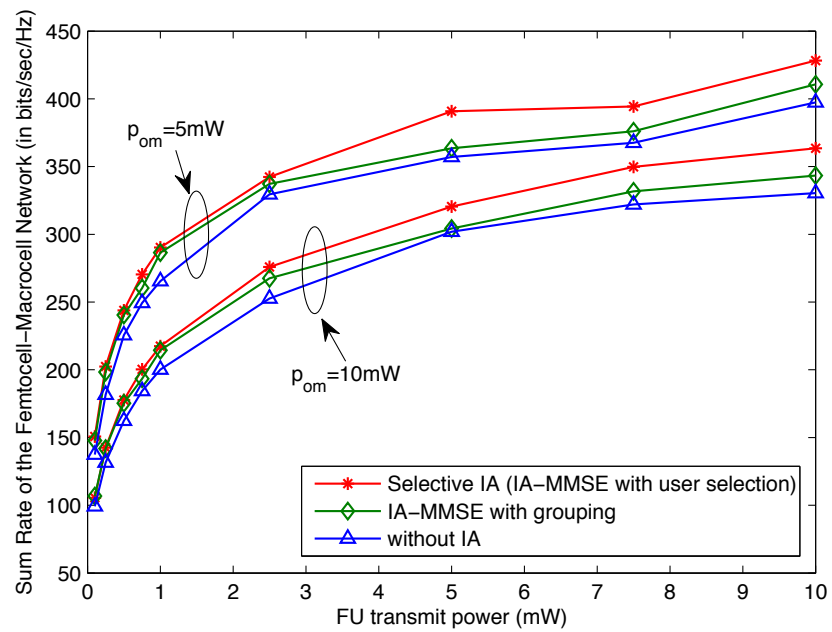

Fig. 9. Sum Rate of the femtocell-macrocell network (in bits/sec/Hz) wrt. FU transmit power.

$p_{f u}=0 \mathrm{dBm} \forall f, u$. We observe that increasing $\tau$ reduces the number of elements in each set for IA, achieving lower leaked interference levels and better IA performance. However, increasing $\tau$ too much results in very few users aligned at each FBS, hindering the benefits of IA. Thus we have empirically chosen $\tau=0.1$ for MU selection. We assume that the number of elements in each set cannot exceed $n=\sum_{f=1}^{F} U_{f}=54$.

Next, we consider the downlink of the first scenario. The convergence of the downlink selective IA algorithm for this system is presented in Fig. 11. The average BER of the MUs are compared for three schemes, selective IA where the set of FBSs for IA at each MU is chosen according to the method proposed in Section VIII, IA with grouping where IA is applied to all FBSs in a femtocell group, and a baseline without IA scheme in Fig. 12. FBS transmit power is given as $1 \mathrm{~mW}$. In the scheme without IA, FBSs apply MMSE precoding for their own users and interference received from the FBSs at the MBS is suppressed by MMSE decoding. Fig. 13 depicts the

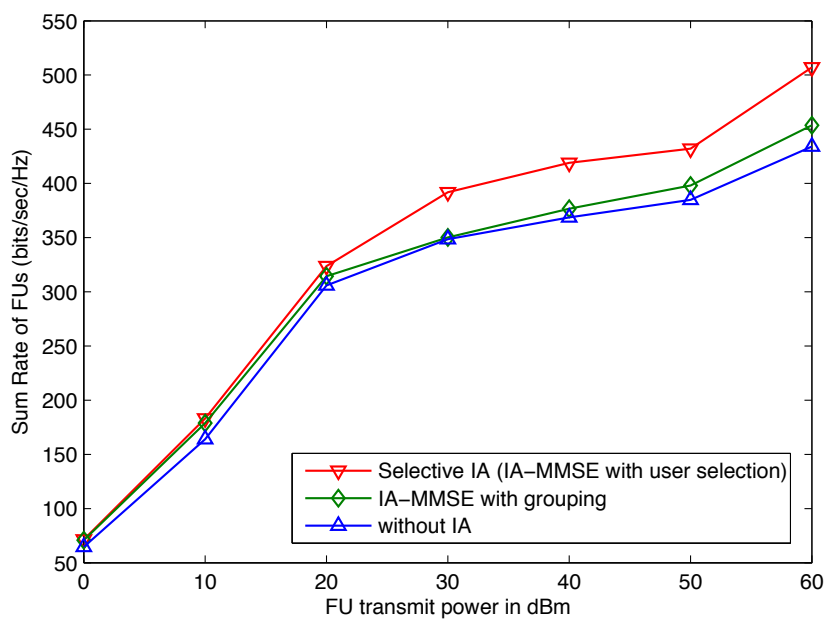

Fig. 10. Sum Rate of the femtocell users (in bits/sec/Hz) wrt. FU transmit power.

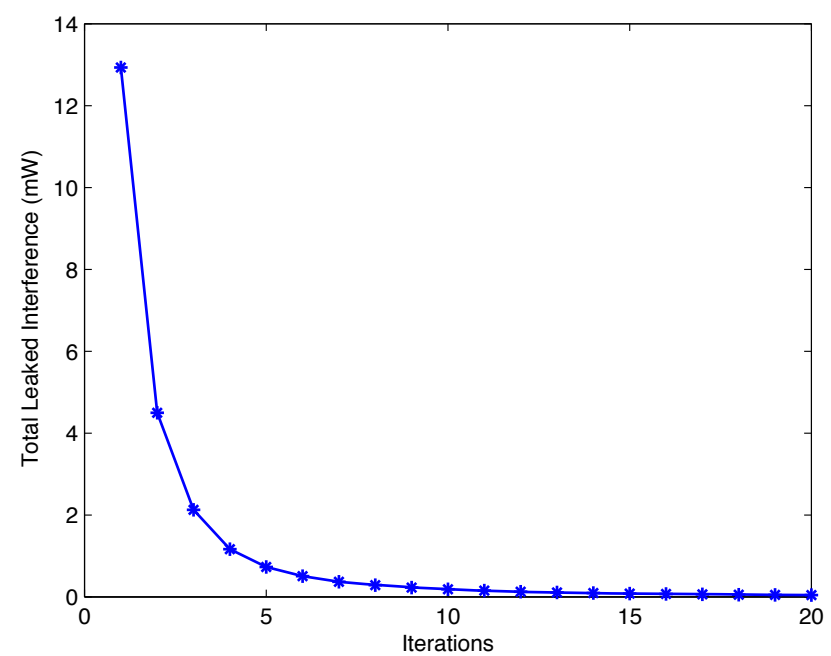

Fig. 11. Convergence of the selective IA algorithm for the downlink.

aggregate sum rates for the downlink with $1 \mathrm{~mW}$ FBS transmit power.

The average BER performance of the MUs for selective IA is presented in Fig. 15(a) for different FBS and MBS transmit powers. Performance of the IA with grouping scheme is given in Fig. 15(b), whereas the performance without IA is presented in Fig. 15(c).

For the second system, we compare the average BERs of the FUs for the selective and grouping schemes as given in Fig. 14, with a maximum transmit power of $1 \mathrm{~W}$ for each mobile user. The selective IA and IA with grouping schemes were also compared to a case where the base stations and the MUs for IA are selected randomly. The simulation results confirm the intuition that the judicious selection of MU for IA is beneficial as compared to these two schemes.

\section{Conclusion}

In this paper, we have considered a cognitive scheme that is applicable to a two-tiered network where the interferers 
TABLE I

EFFECT OF $\tau$ ON SYSTEM PERFORMANCE

\begin{tabular}{|c|c|c|c|}
\hline$\tau$ & $\begin{array}{c}\text { Total Leaked } \\
\text { Interference }\left(\times 10^{-6}\right)\end{array}$ & $\begin{array}{c}\text { Average BER } \\
\left(\times 10^{-3}\right)\end{array}$ & $\begin{array}{c}\text { Aggregate Sum Rate } \\
(\mathrm{bits} / \mathrm{sec} / \mathrm{Hz})\end{array}$ \\
\hline 0.001 & 1.08000 & 7.56 & 211 \\
\hline 0.010 & 0.72100 & 7.50 & 215 \\
\hline 0.025 & 0.37300 & 5.87 & 216 \\
\hline 0.100 & 0.01250 & 2.88 & 218 \\
\hline 0.150 & 0.00610 & 4.19 & 213 \\
\hline 0.250 & 0.00469 & 5.05 & 208 \\
\hline 0.500 & 0.00406 & 8.59 & \\
\hline
\end{tabular}

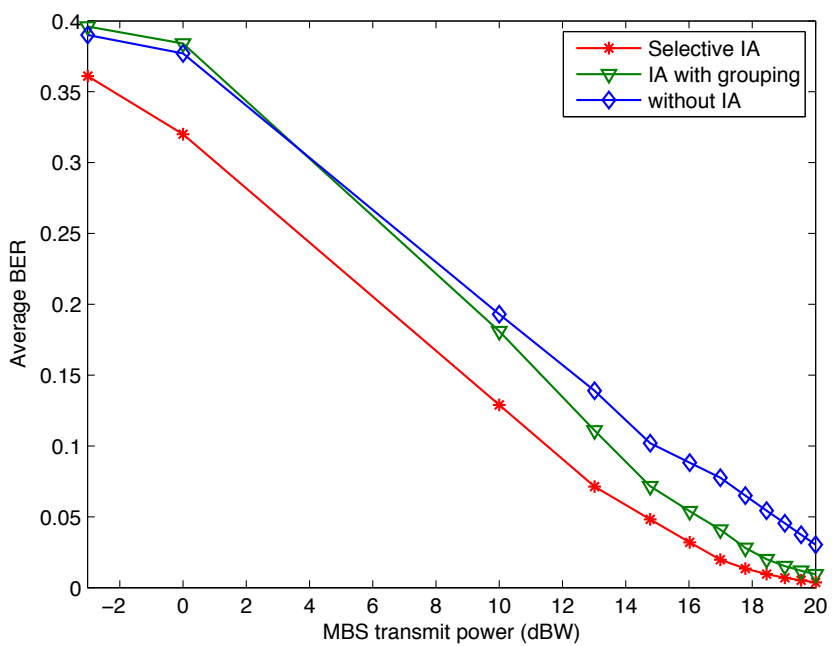

Fig. 12. Average BER of the macrocell users in the downlink wrt. MBS transmit power.

from one tier are distributed over the whole network. We have focused on a heterogeneous system with coexisting cognitive femtocells and a macrocell, and proposed using user selection at the FBSs combined with a distributed IA algorithm to eliminate the destructive uplink macrocell interference at the FBSs. The proposed algorithm is constructed in such a way that is specifically applicable to the tiered network and that it mitigates the problems that may arise from using a centralized IA algorithm, due to backhaul limitations and the excessive load caused on the network. Future work includes considering QoS requirements of the MUs, and designing robust systems with reduced complexity, and incomplete/estimated channel state information.

\section{REFERENCES}

[1] V. Chandrasekhar, J. Andrews, and A. Gatherer, "Femtocell networks: A survey," IEEE Commun. Mag., vol. 46, no. 9, pp. 59-67, Sep. 2008.

[2] Picochip, "The case for home base stations," White Paper, Apr. 2007.

[3] J. Mitola and G. Q. Maguire, "Cognitive radio: Making software radios more personal," IEEE Pers. Commun., vol. 6, pp. 13-18, Aug. 1999.

[4] S. Haykin, "Cognitive radio: Brain-eEmpowered wireless communications," IEEE J. Sel. Areas Commun., vol. 23, pp. 275-283, Feb. 2005.

[5] M. McHenry, E. Livsics, T. Nyugen, and N. Majumdar, "XG dynamic spectrum access field test results," IEEE Commun. Mag., vol. 45, pp. 51-57, June 2007.

[6] Federal Communications Commission, Notice of Inquiry and Notice of Proposed Rulemaking: In the matter of Establishment of an Interefrence Temperature Metric to Quantify and Manage Interference and to Expand Available Unlicensed Operation in Certain Fized, Mobile and Satellite Frequency Bands, ET Docket No. 03-237, Nov. 2003.

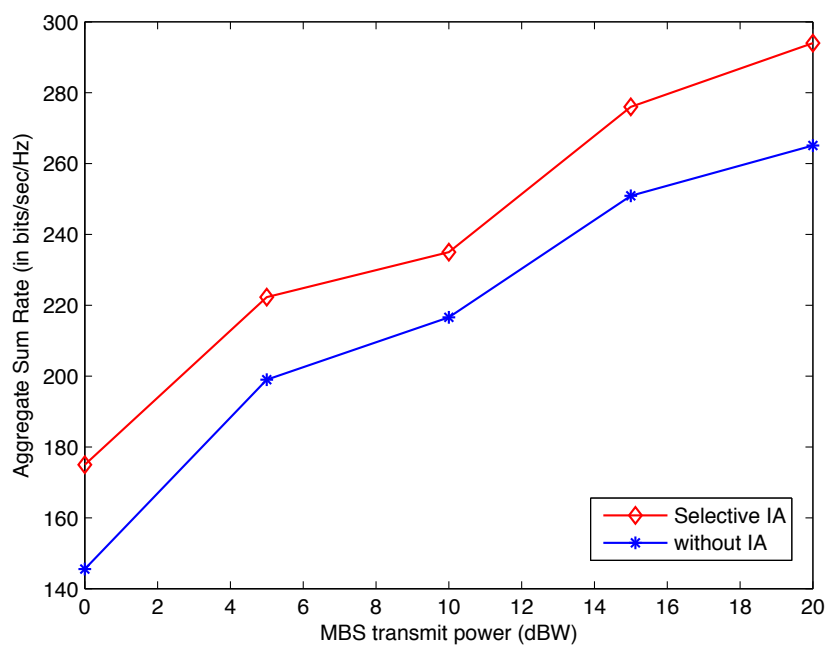

Fig. 13. Aggregate sum rate (in bits/sec/Hz) wrt. MBS transmit power for the downlink.

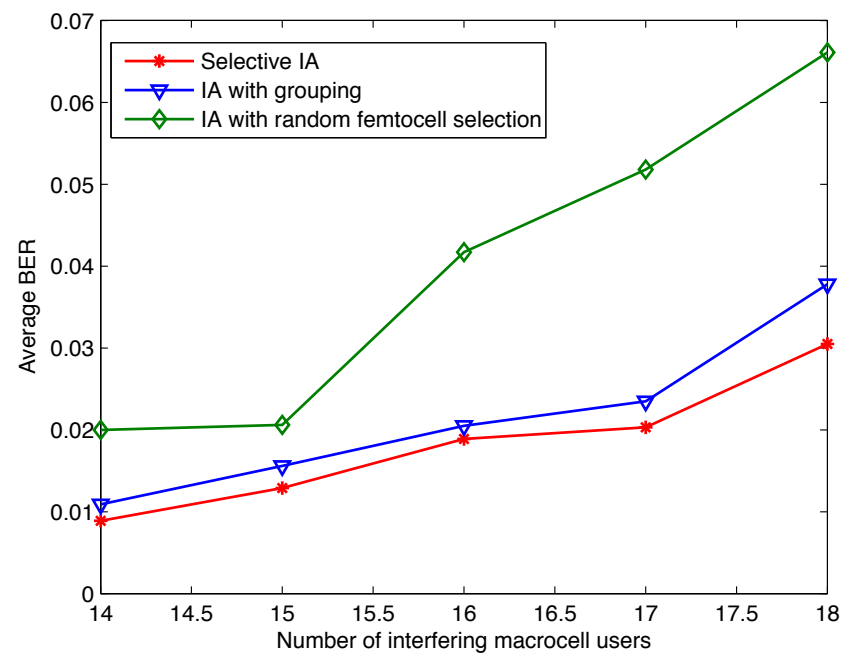

Fig. 14. Average BER of the femtocell users wrt. number of macrocell interferers.

[7] V. D. Chakravarthy, A. K. Shaw, M. A. Temple, and J. P. Stephens, "Cognitive radio - An adaptive waveform with spectral sharing capability," in Proc. IEEE Wireless Commun. Netw. Conf., Mar. 2005, vol. 2, pp. 724-729.

[8] D. Cabric, S. M. Mishra, and R. W. Brodersen, "Implementation issues in spectrum sensing for cognitive radios," in Proc. 38th Asilomar Conf. Signals, Syst. Comput., Nov. 2004, pp. 772-776.

[9] Y. Li, M. Macuha, E. S. Souza, T. Sato, and M. Nanri, "Cognitive interference management in $3 \mathrm{G}$ femtocells," in Proc. IEEE 20th Int. Symp. Pers., Indoor Mobile Radio Commun., Sep. 2009.

[10] A. Adhikary, V. Ntranos, and G. Caire, "Cognitive femtocells: Breaking the spatial reuse barrier of cellular systems," in Proc. Int. Conf. Internet Technol. Appl. (ITA), Feb. 2011.

[11] R. Urgaonkar and M. J. Neely, "Opportunistic cooperation in cognitive femtocell networks," IEEE J. Sel. Areas Commun., Apr. 2012.

[12] V. R. Cadambe and S. A. Jafar, "Interference alignment and degrees of freedom region for the $K$ user interference channel," IEEE Trans. Inf. Theory, vol. 54, no. 8, Aug. 2008.

[13] K. S. Gomadam, V. R. Cadambe, and S. A. Jafar, "Approaching the capacity of wireless networks through distributed interference alignment," arXiv, no. 0803.3816, 2008.

[14] D. A. Schmidt, C. Shi, R. A. Berry, M. L. Honig, and W. Utschick, "Minimum mean squared error interference alignment," in Proc. Asilomar Conf. Signals, Syst., Comput., Nov. 2009, pp. 1106-1110.

[15] S. W. Peters and R. W. Heath Jr., "Interference alignment via alternating 


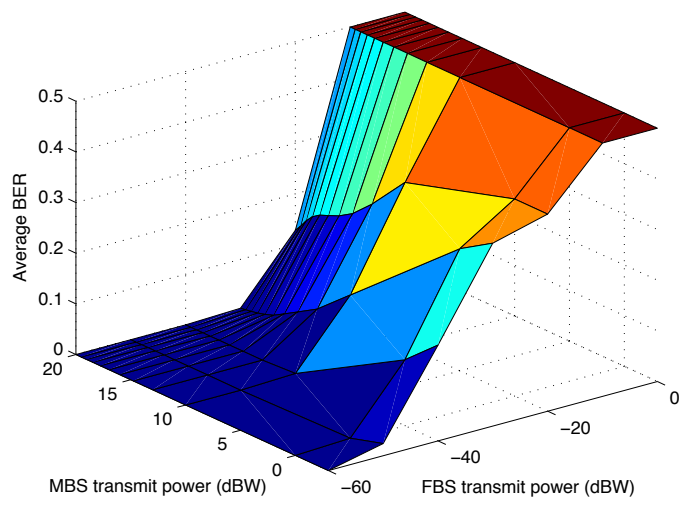

(a) Average BER for selective IA.

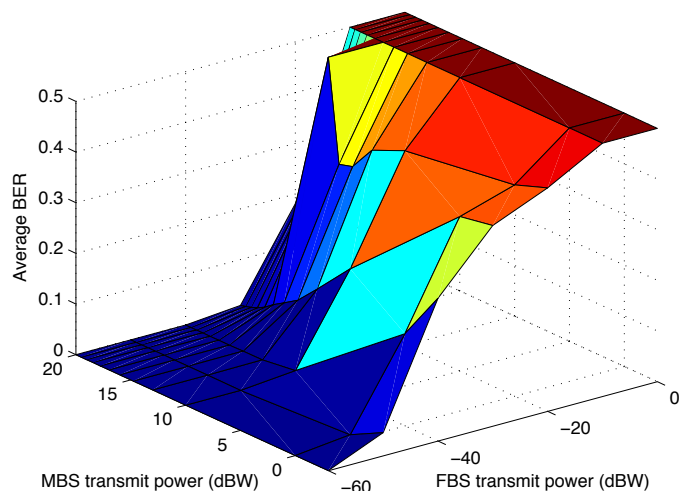

(b) Average BER for IA with grouping.

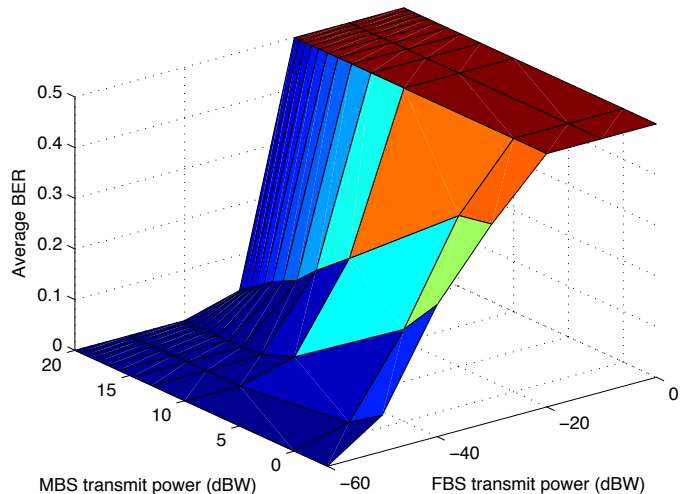

(c) Average BER without IA.

Fig. 15. Average BER comparison of the macrocell users for the downlink

minimization," in Proc. IEEE Int. Conf. Acoust., Speech, Signal Process., Apr. 2009, pp. 2445-2448.

[16] ITU-R Recommendation M.1225, "Guidelines for evaluation of radio transmission technologies for IMT-2000," Feb. 1997.

[17] B. Guler and A. Yener, "Interference alignment for cooperative MIMO femtocell networks," in Proc. IEEE Global Telecom. Conf. (Globecom), Dec. 2011.

[18] H. Lv, T. Liu, X. Hou, and C. Yang, "Adaptive interference alignment for femtocell networks," in Proc. IEEE Int. Conf. Signal Process. (ICSP), Oct. 2010.
[19] F. Pantisano, M. Bennis, W. Saad, and M. Debbah, "Cooperative interference alignment in femtocell networks," in Proc. IEEE Global Telecom. Conf. (Globecom), Dec. 2011.

[20] H. Huang and V. Lau, "Partial interference alignment for $K$-user MIMO interference channels," IEEE Trans. Signal Process., vol. 59, no. 10, pp. 4900-4908, Oct. 2011.

[21] H. Yu and Y. Sung, "Least squares approach to joint beam design for interference alignment in multiuser multi-input multi-output interference channels," IEEE Trans. Signal Process., vol. 58, no. 9, Sep. 2010.

[22] S. Boyd and L. Vandenberghe, Convex Optimization. Cambridge University Press, 2004.

[23] S. Serbetli and A. Yener, "Transceiver optimization for multiuser MIMO systems," IEEE Trans. Signal Process., vol. 52, no.1, pp. 214-226, Jan. 2004.

[24] S. Ulukus and A. Yener, "Iterative transmitter and receiver optimization for CDMA networks," IEEE Trans. Wireless Commun., vol. 3, no. 6 , pp. 1974-1979, Nov. 2004

[25] C. M. Yetis, T. Gou, S. A. Jafar, and A. H. Kayran, "On feasibility of interference alignment in MIMO interference networks," IEEE Trans. Signal Process., vol. 58, no. 9, pp. 4771-4782, Sep. 2010.

[26] D. A. Cox, J. B. Little, and D. B. O'Shea, Using Algebraic Geometry, 2nd ed. New York, N.Y: Springer, 2005.

[27] G. Bresler, D. Cartwright, and D. Tse, "Settling the feasibility of interference alignment for the MIMO interference channel: The symmetric square case," in Proc. 13th Int. Telecommun. Week Conf. (ITW), 2011.

[28] M. Razaviyayn, G. Lyubeznik, and Z.-Q. Luo, "On the degrees of freedom achievable through interference alignment in a MIMO interference channel," IEEE Trans. Signal Process., vol. 60, no. 2, pp. 812-821, 2012.

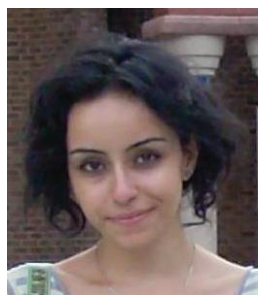

Basak Guler received her B.Sc. degree in electrical and electronics engineering from Middle East Technical University (METU), Ankara, Turkey, in 2009 and her M.Sc. degree in electrical engineering from the Wireless Communications and Networking Laboratory, Pennsylvania State University, University Park, PA, in 2012. She is currently pursuing the Ph.D. degree with the Wireless Communications and Networking Laboratory, Pennsylvania State University. Her research interests include interference management in heterogeneous wireless networks, femtocell-macrocell communication systems, and semantic source coding.

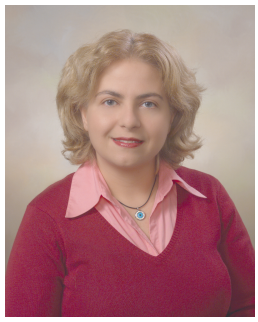

Aylin Yener (S'91-M'00) received the B.Sc. degree in electrical and electronics engineering, and the B.Sc. degree in physics, from Bogazici University, Istanbul, Turkey. She also received the the M.S. and $\mathrm{Ph} . \mathrm{D}$. degrees in electrical and computer engineering from the Wireless Information Network Laboratory (WINLAB), Rutgers University, New Brunswick, NJ. For three semesters in fall 2000 she was the P.C. Rossin Assistant Professor in the Electrical Engineering and Computer Science Department of Lehigh University, PA. In 2002, she joined the faculty of The Pennsylvania State University, University Park, PA, where she was an Assistant Professor, then Associate Professor, and since 2010, is a Professor of electrical engineering. During the academic year 2008-2009, she was a Visiting Associate Professor with the Department of Electrical Engineering, Stanford University, CA. Her research interests are in information theory, communication theory, and network science, with recent emphasis on green communications and information security. She received the NSF CAREER award in 2003

Dr. Yener has served as a technical program chair or co-chair for various conferences for the IEEE Communications Society, as an associate editor for the IEEE TRANSACTIONS ON COMMUNICATIONS, and as an associate editor and editorial advisory board member for the IEEE TRANSACTIONS ON WiRELESS COMmUNiCATIONS. She served as the student committee chair for the IEEE Information Theory Society from 2007-2011 and was the cofounder of the Annual School of Information Theory in North America, coorganizing the school in 2008, 2009, and 2010. Dr. Yener currently serves on the board of governors of the IEEE Information Theory Society as its treasurer. 